serving $>400$ patients, to establish prevalence and potential risk factors for infection.

Methods Sputum samples were obtained from 100 randomly selected CF outpatients and sent for routine microbiology and PJ DNA PCR assay at enrolment, subsequent visits, and pulmonary exacerbations requiring intravenous antibiotics within 4 months. Data were recorded for demographics, co-morbidities symptom score, spirometry, inflammatory markers, and prophylactic or recent therapeutic antibiotic therapy. Univariate comparisons were made between sputum PJ positive and negative patients. Chi square tests were used for categorical comparisons and independent sample t-tests for continuous independent variables.

Results Of the 100 patients, 4 of 100 had a positive sputum PJ PCR at baseline. 50 patients had a routine follow up sample between 1 and 4 months: 2 were positive for PJ. 22 patients had a sputum sample analysed at the onset of a pulmonary exacerbation of which 1 was positive for PJ. Hence, a total of 7 of 100 patients had a single positive sample by PCR for PJ. No patient has had $>1$ positive sample. None of the baseline parameters were significantly different between PJ positive and PJ negative patients at the level $\mathrm{p}$

Conclusion These results suggest that PJ is not an important infecting pathogen in this UK cohort of CF patients. This may be due to frequent use co-trimoxazole for pulmonary exacerbations and high prevalence of prophylactic macrolide antibiotic therapy at our centre compared to other published studies. These preliminary data were underpowered to accurately compare baseline characteristics between PJ positive and PJ negative patients.

\section{P202 STUDYING THE RELATIONSHIP BETWEEN MATRIX METALLOPROTEINASES AND LUNG TISSUE DAMAGE DURING A CLINICAL EXACERBATION OF CYSTIC FIBROSIS}

${ }^{1} \mathrm{HAP}$ Passman, ${ }^{2} \mathrm{TD}$ Daniels, ${ }^{2} \mathrm{PE}$ Elkington. 'University of Southampton Faculty of Medicine, Southampton, UK; ${ }^{2}$ University Hospital Southampton NHS Foundation Trust, Southampton, UK

\subsection{6/thoraxjnl-2014-206260.331}

Background Cystic Fibrosis (CF) is the commonest fatal autosomal recessive condition of Caucasians. The lung in CF is characterised by chronic bacterial colonisation, inflammation, and lung tissue destruction. Matrix Metalloproteinases (MMPs) are proteases that are expressed in inflammatory states that degrade lung matrix. Whilst MMPs are known to be raised in many inflammatory lung conditions, their role in CF is poorly understood.

Aims The study aimed to compare lung function and MMP levels throughout an exacerbation.

Methods Sputum samples and clinical information were obtained with written consent from 23 adult subjects with CF. Samples were collected at day 0, day 14 and week 6 of an exacerbation of CF. Samples were frozen, before Luminex bioassay and IL-8 ELISA was performed. The sputum concentration of MMP 1, 2, 3, 7, 8, 9,10,12,13 and IL-8 were measured.

Results A statistically significant increase in IL-8 concentration $(\mathrm{pg} / \mathrm{ml})$ occurred between day 14 and week $6(\mathrm{p}=0.0387)$. The concentration of MMP-9 decreased from day 0 to day 14 ( $\mathrm{p}=$ 0.006), and then rose significantly between day 14 and week 6 $(\mathrm{p}=0.0412)$. However, the concentration of MMP-9 at week 6 was significantly less than at day 0 (0.0453). A significant decrease in the concentration of MMP-8 occurred between day

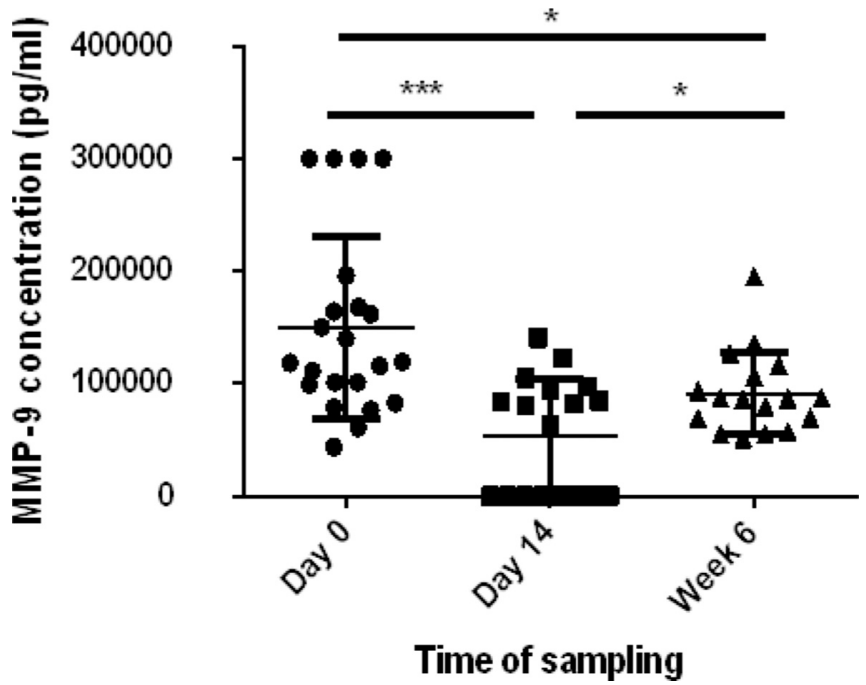

Abstract P202 Figure 1 The sputum concentration of MMP-9 (pg/ml) at sample time. Values at 3000 represent values above the level of detection. Significant values are represented with a line and an asterix ${ }^{*}$ ). A significant decrease in MMP-9 concentration was found between Day 0 and Day $14(p=0.006)$. An increase in MMP-9 concentration was seen between day 14 and week $6(p=0.0412)$. There was a statistically significant decrease in MMP-9 concentration between day 0 and week $6(p=0.0453)$

0 and day $14(\mathrm{p}=0.0124)$; and between day 0 to week $6(\mathrm{p}=$ 0.0426).

Conclusions MMPs and other inflammatory markers are raised during exacerbations, and fall with treatment. High proteolytic activity may lead to a worsening lung function and contribute significantly to structural changes within the CF lung. Furthering our understanding of this diverse group of proteases could lead to potential novel therapeutic targets which could help prevent irreversible lung damage.

\section{P203 DEVELOPMENT OF AN OPTIMAL F/HN PSEUDOTYPED SIV VECTOR FOR CF GENE THERAPY}

${ }^{1}$ SC Hyde, ${ }^{1}$ Ewfw Alton, 'AC Boyd, ${ }^{1} \mathrm{MM}$ Connolly, ${ }^{1} \mathrm{M}$ Chan, ${ }^{1} \mathrm{JC}$ Davies, 'LA Davies, ${ }^{1} \mathrm{~S}$ Gea-Sorli, ${ }^{1} \mathrm{U}$ Griesenbach, ${ }^{2} \mathrm{M}$ Hasegawa, ${ }^{1} \mathrm{JA}$ Innes, ${ }^{2} \mathrm{M}$ Inoue, ${ }^{1} \mathrm{G}$ McLachlan, ${ }^{1} \mathrm{C}$ Meng, ${ }^{1}$ IA Pringle, 'SG Sumner-Jones, ${ }^{2}$ SG Tsugumine, 'DR Gill. ' UK Cystic Fibrosis Gene Therapy Consortium, Oxford, London and Edinburgh, UK; ${ }^{2}$ DNAVEC Corporation, Tsukuba, Japan

\subsection{6/thoraxjnl-2014-206260.332}

We are developing lung gene transfer vectors to treat acquired and inherited lung disorders such as cystic fibrosis, and have identified two platforms for efficient respiratory gene delivery: one non-viral system based on CpG-free plasmid DNA combined with cationic lipids (pDNA/GL67A), which has recently completed evaluation in a Phase IIb clinical study; and one novel viral system based on a recombinant simian immunodeficiency virus pseudotyped with the $\mathrm{F} / \mathrm{HN}$ proteins of Sendai virus (rSIV. $\mathrm{F} / \mathrm{HN}$ ) to promote airway cell uptake. Here we report on the development of a "third generation" rSIV. F/HN vector suitable for use in the clinic. The vector is manufactured by transient transfection of cultured human cells using five producer plasmids, all of which have been engineered to be pharmacopoeia compliant. A variety of vector configurations, including a range of enhancers/promoters and transgenes, were evaluated in a panel of airway models. rSIV. F/HN vectors directed high-level, robust gene expression in fully differentiated human airway cells, 
human nasal brushings and human and sheep lung slices. In the mouse nose and lung, the preferred configuration directed up to $\mathrm{x} 500$-fold higher transgene expression than the non-viral platform, for the lifetime of the animal. Transgene expression was observed in $14.1 \%$ of lung epithelial cells ( $p<0.0001$ compared to controls). Repeated monthly administration (3X) was possible without loss of expression or significant histological inflammatory reactivity. Reassuringly, insertion site profiling from transduced cell lines and mouse nose/lung samples reveals a pattern of integration comparable to those reported for other lentiviral vectors in clinical development, with no evidence for enrichment of insertion at undesirable loci. The stability of rSIV. F/HN vectors was evaluated in two bronchoscope catheters and two aerosol generation devices. Encouragingly for clinical translation, no significant loss of transduction activity was noted with any of these clinically relevant delivery devices $(\mathrm{p}=0.64)$. Delivery of rSIV. F/HN expressing CFTR to sheep lung resulted in CFTR mRNA at $\sim 30 \%$ the levels of endogenous ovine CFTR ( $p<$ 0.0001 compared to non-treated lobes), exceeding presumed therapeutic levels. With the majority of translational hurdles addressed, we are now entering toxicology studies and the final stages of pharmaceutical development prior to entering clinical trials.

\section{P204 IMMUNE RESPONSES TO SINGLE AND REPEATED ADMINISTRATION OF PGM169/GL67A: THE UK CF GENE THERAPY CONSORTIUM CLINICAL TRIALS}

${ }^{1} \mathrm{U}$ Griesenbach, ${ }^{1} \mathrm{AC}$ Boyd, ${ }^{2} \mathrm{R}$ Calcedo, ${ }^{3} \mathrm{~S}$ Cheng, ${ }^{1} \mathrm{~S}$ Cunningham, ${ }^{1} \mathrm{JC}$ Davies, ${ }^{1} \mathrm{M}$ Dewar, ${ }^{1}$ DR Gill, ${ }^{1} \mathrm{~A}$ Doherty, ${ }^{1} \mathrm{~T}$ Higgins, ${ }^{1} \mathrm{SC}$ Hyde, ${ }^{1} \mathrm{M}$ Manvell, ${ }^{1} \mathrm{C}$ Meng, ${ }^{1} \mathrm{JA}$ Innes, ${ }^{2} \mathrm{MP}$ Limberis, ${ }^{1} E$ Punch, ${ }^{3} \mathrm{R}$ Scheule, ${ }^{1} \mathrm{~N}$ Soussi, 'S Soussi, ${ }^{2} \mathrm{JM}$ Wilson, ${ }^{1}$ Ewfw Alton. ${ }^{1}$ UK Cystic Fibrosis Gene Therapy Consortium, London, Oxford, Edinburgh, UK; ' ${ }^{2}$ ene Therapy Program, Department of Pathology and Laboratory Medicine, University of Pennsylvania., Pennsylvania., USA; ${ }^{3}$ Genzyme-Sanofi, MA, USA

\subsection{6/thoraxjn-2014-206260.333}

Although most CF patients express CFTR protein (albeit mutant) and should therefore not recognise the wild-type CFTR protein as foreign, there is an inherent risk of activation of T-cells against the recombinant wild-type protein after gene therapy. In addition, we have previously shown that approximately 10\% of $\mathrm{CF}$ and non-CF subjects carry self-reactive CFTR-specific T-cells (Calcedo et al, Hum Gene Ther Clin Dev 2013). The reason for this is unknown and it is also unclear whether being positive for self-reactive T-cells affects disease severity or increases the risk of further T-cell activation after gene therapy.

As part of the UKCFGTC Phase I/IIa Pilot study [in which patients received a single dose $(5,10$ or $20 \mathrm{mls})$ of the non-viral formulation pGM169/GL67A] peripheral blood mononuclear cells (PBMC) were collected prior to dosing and approximately 4 weeks after nebulisation of $5 \mathrm{ml}(\mathrm{n}=2), 10 \mathrm{ml}(\mathrm{n}=6)$ or 20 $\mathrm{ml}(\mathrm{n}=17)$ of pGM169/GL67A. IFN-g ELISPOT to detect CFTR-specific T-cells in PBMC was performed. CFTR-specific T-cells were detectable in one patient pre- and post-dosing. In the remaining 18 patients we did not detect CFTR-specific Tcells. In addition we quantified anti-DNA antibodies (antinuclear and anti-cytoplasmic) in blood samples taken pre- and approximately 4 weeks post-dosing $(\mathrm{n}=7(5 \mathrm{ml}), \mathrm{n}=10$ $(10 \mathrm{ml})$ and $\mathrm{n}=17(20 \mathrm{ml})$. We did not observe any evidence for induction of anti-DNA antibodies after a single dose of pGM169/GL67A.

The UKCFGTC has now completed a Phase IIb multi-dose clinical trial in May 2014 (ClinicalTrials.gov identification number - NCT01621867). CF patients received 12 monthly doses of pGM169/GL67A (115 completed nine or more doses), or placebo by aerosol. PBMC were collected on two occasions prior to dose 1 to establish baseline levels for CFTR-specific Tcells, approximately 4 weeks after Dose 4 or 5 , and 2 to 4 weeks after Dose 12 and the ELISPOT was performed. In addition antiDNA antibodies were quantified. The Phase IIb trial will be unblinded in Summer 2014 to allow data analysis and all data will be presented at the conference.

Funded by the NIHR/EME Programme and the Cystic Fibrosis Trust.

\section{Lung function testing: new approaches}

\section{P205 MULTIPLE BREATH WASHOUTS IN CHILDREN CAN BE SIGNIFICANTLY SHORTENED WITHOUT COMPROMISING MEASUREMENT QUALITY}

${ }^{1} \mathrm{FA}$ Ahmad, ${ }^{2} \mathrm{SI}$ Inving, ${ }^{2} \mathrm{AB}$ Bush, ${ }^{2} \mathrm{LF}$ Fleming, ${ }^{2} \mathrm{SS}$ Saglani. ${ }^{1}$ Imperial College, London, UK; ${ }^{2}$ Royal Brompton and Harefield NHS Foundation Trust and Imperial College, London, UK

\subsection{6/thoraxjn-2014-206260.334}

Background Multiple-breath washout (MBW) is used to calculate a measure of ventilation heterogeneity, the lung clearance index (LCI), and requires tidal breathing until a previously inspired tracer gas concentration falls below 1/40th of the initial value, an arbitrary threshold. LCI is usually performed in triplicate, each taking 4-8 min to complete which may be taxing, particularly in young children and those with marked airflow obstruction. Shortened LCI is of interest since a reduction in the test time may increase feasibility and improve the clinical applicability of the measurement.

We hypothesised that LCI measurements could be reliably shortened. We also investigated whether shortened MBW was responsive to an intervention.

Patients and methods We calculated LCI from a fixed time point, and from a fixed number of breaths, as well as LCI and $25 \%$ (LCI0.25), 50\% (LCI0.5) and 75\% (LCI0.75) of 1/40th of the initial concentration of tracer gas (LCIstd) and the time saved, in children aged 6-16 years with asthma $(\mathrm{n}=21)$, cystic fibrosis $(\mathrm{CF}, \mathrm{n}=20)$ and primary ciliary dyskinesia (PCD, $\mathrm{n}=19)$, and healthy controls $(\mathrm{n}=17)$, aged $3-18$ years. Shortened LCI was also calculated in 29 asthmatic children pre and one month post one intra-muscular triamcinolone injection, part of our clinical severe asthma protocol.

Results Calculating shortened LCI from a fixed washout time or breath number was not reliable. However, all shortened LCI measurements from initial gas concentration correlated significantly with LCIstd in each disease group. LCI0.5 presented a balance between correlation with LCIstd (see figure) and timesaving. Mean proportion of time saved per washout, using LCI0.5, was 27\% (asthma), 28\% (CF) and 31\% (PCD). Furthermore, LCI0.5 was significantly reduced after triamcinolone in children with severe asthma (mean LCI0.5 pre, 5.5 and 5.1 post triamcinolone, $p=0.02$ ), and the change was similar to that demonstrated using LCIstd (mean LCIstd pre, 7.8 and post 7.0, $\mathrm{p}=0.001$ ).

Conclusion We show for the first time that LCI measurements can be shortened without loss of information in school-children with asthma, CF and PCD. LCI0.5 was the optimal surrogate measure for LCIstd when proportion of time saved, correlation 